\title{
A DOUBLE INEQUALITY FOR REMAINDER OF POWER SERIES OF TANGENT FUNCTION
}

\author{
CHAO-PING CHEN AND FENG QI
}

\begin{abstract}
By mathematical induction, an identity and a double inequality for remainder of
\end{abstract} power series of tangent function are established.

\section{Introduction}

It is well known that Bernoulli numbers $B_{i}$ are defined [11] by

$$
\frac{x}{e^{x}-1}=1-\frac{1}{2} x+\sum_{i=1}^{\infty}(-1)^{i+1} \frac{B_{i}}{(2 i) !} x^{2 i}, \quad|x|<2 \pi .
$$

About Bernoulli numbers, some new results can be found in $[1,3,5]$.

The tangent and cotangent can be expanded into power series with coefficients involving Bernoulli numbers as follows [11, p.5]:

$$
\begin{array}{ll}
\tan x=\sum_{i=1}^{\infty} \frac{2^{2 i}\left(2^{2 i}-1\right) B_{i}}{(2 i) !} x^{2 i-1}, & |x|<\frac{\pi}{2} \\
\cot x=\frac{1}{x}-\sum_{i=1}^{\infty} \frac{2^{2 i} B_{i}}{(2 i) !} x^{2 i-1}, & |x|<\pi
\end{array}
$$

Introduce two notations $S_{n}(x)$ and $r_{n}(x)$ by

$$
\begin{aligned}
& S_{n}(x)=\sum_{i=1}^{n} \frac{2^{2 i}\left(2^{2 i}-1\right) B_{i}}{(2 i) !} x^{2 i-1}, \\
& r_{n}(x)=\tan x-S_{n}(x)
\end{aligned}
$$

Received May 17, 2002; revised July 15, 2002.

2000 Mathematics Subject Classification. 26D15.

Key words and phrases. Tangent function, a double inequality, power series, remainder, Bernoulli number.

The authors were supported in part by NNSF (\#10001016) of China, SF for the Prominent Youth of Henan Province, SF of Henan Innovation Talents at Universities, Doctor Fund of Jiaozuo Institute of Technology, P.R. China. 
for $0<x<\frac{\pi}{2}$. Then $\tan x=\lim _{n \rightarrow \infty} S_{n}(x)$. We call $r_{n}(x)$ the remainder of power series for tangent function.

For elementary functions $\sin x, \cos x$, and $e^{x}$, there are much literature on estimates of their remainder. For examples, see [6, 7, 9]. The methods used in $[6,7,9]$ have been applied to construct inequalities of elliptic integrals. See $[8,10]$. Some inequalities involving $\tan x$ were researched by the second author and others in [2].

In this article, we will establish a double inequality for remainder $r_{n}(x)$ of power series for $\tan x$. That is

Theorem 1. For $x \in\left(0, \frac{\pi}{2}\right)$ and $n \in \mathbb{N}$, we have

$$
\frac{2^{2(n+1)}\left(2^{2(n+1)}-1\right) B_{n+1}}{(2 n+2) !} x^{2 n} \tan x<\tan x-S_{n}(x)<\left(\frac{2}{\pi}\right)^{2 n} x^{2 n} \tan x .
$$

Remark 1. If taking $n=1$ in (6), we have for $x \in(0,1)$

$$
\frac{\pi}{2} \cdot \frac{x}{1-\frac{7 \pi^{2}}{360} x^{2}}<\tan \frac{\pi x}{2}<\frac{\pi}{2} \cdot \frac{x}{1-x^{2}} .
$$

For $0<x<\frac{3}{\pi} \sqrt{\frac{5\left(\pi^{2}-8\right)}{38}}$, the left inequality in (7) is better than the left inequality in the following Becker-Stark inequality [4, p.351]:

$$
\frac{4}{\pi} \cdot \frac{x}{1-x^{2}}<\tan \frac{\pi x}{2}<\frac{\pi}{2} \cdot \frac{x}{1-x^{2}}, \quad x \in(0,1) .
$$

If taking $n=2$ in (6), we obtain

$$
x+\frac{1}{3} x^{3}+\frac{2}{15} x^{4} \tan x<\tan x<x+\frac{1}{3} x^{3}+\left(\frac{2}{\pi}\right)^{4} x^{4} \tan x, \quad x \in\left(0, \frac{\pi}{2}\right) .
$$

The constants $\frac{2}{15}$ and $\left(\frac{2}{\pi}\right)^{4}$ in (9) are best possible.

For $x \in\left(0, \frac{\pi}{6}\right)$, the Djokvie inequality states $[4$, p.350] that

$$
x+\frac{1}{3} x^{3}<\tan x<x+\frac{4}{9} x^{3} .
$$

Since

$$
\frac{1}{3}+\left(\frac{2}{\pi}\right)^{4} x \tan x<\frac{1}{3}+\left(\frac{2}{\pi}\right)^{4} \cdot \frac{\pi}{6} \cdot \frac{1}{\sqrt{3}}<\frac{4}{9},
$$

thus, the inequality in (9) is better than those in (10).

\section{Proof of Theorem}

Let

$$
h_{n}(x)=\frac{\tan x-S_{n}(x)}{x^{2 n} \tan x}
$$


for $n \in \mathbb{N}$. Then we have the following lemma.

Lemma 1. For $x \in\left(0, \frac{\pi}{2}\right)$ and $n \in \mathbb{N}$, we have

$$
h_{n}(x)=\sum_{j=1}^{n} \frac{2^{2(n-j+1)}\left[2^{2(n-j+1)}-1\right] B_{n-j+1}}{[2(n-j+1)] !} \sum_{k=j}^{\infty} \frac{2^{2 k} B_{k}}{(2 k) !} x^{2(k-j)} .
$$

Proof. We shall prove this lemma by mathematical induction on $n$.

For $n=1$, we have

$$
\begin{aligned}
h_{1}(x) & =\frac{\tan x-S_{1}(x)}{x^{2} \tan x} \\
& =\frac{1}{x^{2}}-\frac{\cot x}{x} \\
& =\frac{1}{x^{2}}-\frac{1}{x}\left(\frac{1}{x}-\sum_{k=1}^{\infty} \frac{2^{2 k} B_{k}}{(2 k) !} x^{2 k-1}\right) \\
& =\sum_{k=1}^{\infty} \frac{2^{2 k} B_{k}}{(2 k) !} x^{2(k-1)},
\end{aligned}
$$

the formula (12) holds for $n=1$.

For $n=2$, we have

$$
\begin{aligned}
h_{2}(x) & =\frac{\tan x-S_{2}(x)}{x^{4} \tan x} \\
& =\frac{1}{x^{4}}-\frac{\cot x}{x^{3}}-\frac{\cot x}{3 x} \\
& =\frac{1}{x^{4}}-\frac{1}{x^{3}}\left(\frac{1}{x}-\frac{1}{3} x-\sum_{k=2}^{\infty} \frac{2^{2 k} B_{k}}{(2 k) !} x^{2 k-1}\right)-\frac{1}{3 x}\left(\frac{1}{x}-\sum_{k=1}^{\infty} \frac{2^{2 k} B_{k}}{(2 k) !} x^{2 k-1}\right) \\
& =\sum_{k=2}^{\infty} \frac{2^{2 k} B_{k}}{(2 k) !} x^{2(k-1)}+\sum_{k=1}^{\infty} \frac{2^{2 k} B_{k}}{3 \cdot(2 k) !} x^{2(k-1)},
\end{aligned}
$$

the formula (12) holds for $n=2$.

Assume formula (12) holds for $n=m$. Then for $n=m+1$, we have

$$
\begin{aligned}
h_{m+1} & =\frac{\tan x-S_{m+1}(x)}{x^{2(m+1)} \tan x} \\
& =\frac{\tan x-S_{m}(x)-\frac{2^{2(m+1)}\left(2^{2(m+1)}-1\right) B_{m+1}}{[2(m+1)] !} x^{2 m+1}}{x^{2(m+1)} \tan x} \\
& =\frac{1}{x^{2}} \cdot \frac{\tan x-S_{m}(x)}{x^{2 m} \tan x}-\frac{2^{2(m+1)}\left(2^{2(m+1)}-1\right) B_{m+1}}{[2(m+1)] !} \cdot \frac{\cot x}{x} \\
& =\frac{1}{x^{2}} \sum_{j=1}^{m} \frac{2^{2(m-j+1)}\left[2^{2(m-j+1)}-1\right] B_{m-j+1}}{[2(m-j+1)] !} \sum_{k=j}^{\infty} \frac{2^{2 k} B_{k}}{(2 k) !} x^{2(k-j)}
\end{aligned}
$$




$$
\begin{aligned}
& -\frac{2^{2(m+1)}\left(2^{2(m+1)}-1\right) B_{m+1}}{[2(m+1)] !} \cdot \frac{1}{x}\left(\frac{1}{x}-\sum_{k=1}^{\infty} \frac{2^{2 k} B_{k}}{(2 k) !} x^{2 k-1}\right) \\
= & \frac{1}{x^{2}} \sum_{j=1}^{m} \frac{2^{2 j}\left(2^{2 j}-1\right) B_{j}}{(2 j) !} \cdot \frac{2^{2(m-j+1)} B_{m-j+1}}{[2(m-j+1)] !} \\
& +\sum_{j=1}^{m+1} \frac{2^{2(m-j+2)}\left(2^{2(m-j+2)}-1\right) B_{m-j+2}}{[2(m-j+2)] !} \sum_{k=j}^{\infty} \frac{2^{2 k} B_{k}}{(2 k) !} x^{2(k-j)} \\
& -\frac{2^{2(m+1)}\left[2^{2(m+1)}-1\right] B_{m+1}}{[2(m+1)] !} \frac{1}{x^{2}}+\frac{2^{2(m+1)}\left[2^{2(m+1)}-1\right] B_{m+1}}{[2(m+1)] !} \sum_{k=1}^{\infty} \frac{2^{2 k} B_{k}}{(2 k) !} x^{2(k-1)} \\
= & \sum_{j=1}^{m+1} \frac{2^{2(m-j+2)}\left[2^{2(m-j+2)}-1\right] B_{m-j+2}}{[2(m-j+2)] !} \sum_{k=j}^{\infty} \frac{2^{2 k} B_{k}}{(2 k) !} x^{2(k-j)} \\
& +\frac{1}{x^{2}} \sum_{j=1}^{m} \frac{2^{2 j}\left(2^{2 j}-1\right) B_{j}}{(2 j) !} \cdot \frac{2^{2(m-j+1)} B_{m-j+1}}{[2(m-j+1)] !}-\frac{2^{2(m+1)}\left(2^{2(m+1)}-1\right) B_{m+1}}{[2(m+1)] !} \cdot \frac{1}{x^{2}} .
\end{aligned}
$$

Since $\tan x \cot x=1$, we have

$$
\left(\sum_{i=1}^{\infty} \frac{2^{2 i}\left(2^{2 i}-1\right) B_{i}}{(2 i) !} x^{2 i-1}\right)\left(\frac{1}{x}-\sum_{i=1}^{\infty} \frac{2^{2 i} B_{i}}{(2 i) !} x^{2 i-1}\right)=1,
$$

which is equivalent to

$$
\sum_{i=2}^{\infty} \frac{2^{2 i}\left(2^{2 i}-1\right) B_{i}}{(2 i) !} x^{2 i-2}=\left[\sum_{i=1}^{\infty} \frac{2^{2 i}\left(2^{2 i}-1\right) B_{i}}{(2 i) !} x^{2 i-1}\right] \sum_{i=1}^{\infty} \frac{2^{2 i} B_{i}}{(2 i) !} x^{2 i-1},
$$

equating coefficients of the term $x^{2 m}$ on both sides of (14) yields

$$
\frac{2^{2(m+1)}\left(2^{2(m+1)}-1\right) B_{m+1}}{(2(m+1)) !}=\sum_{j=1}^{m} \frac{2^{2 j}\left(2^{2 j}-1\right) B_{j}}{(2 j) !} \cdot \frac{2^{2(m-j+1)} B_{m-j+1}}{[2(m-j+1)] !} .
$$

Substituting (15) into (13) and simplifying gives us

$$
h_{m+1}(x)=\sum_{j=1}^{m+1} \frac{2^{2(m-j+2)}\left(2^{2(m-j+2)}-1\right) B_{m-j+2}}{[2(m-j+2)] !} \sum_{k=j}^{\infty} \frac{2^{2 k} B_{k}}{(2 k) !} x^{2(k-j)} .
$$

By induction, the proof of Lemma 1 is complete.

Now we give a proof of Theorem 1 .

Proof of Theorem 1. From (12), it is deduced that $h_{n}^{\prime}(x)>0$, and $h_{n}(x)$ is strictly increasing in $\left(0, \frac{\pi}{2}\right)$, Easy computing yields

$$
h_{n}(0+0)=\frac{2^{2 n+2}\left(2^{2 n+2)}-1\right) B_{n+1}}{(2 n+2) !},
$$




$$
h\left(\frac{\pi}{2}-0\right)=\left(\frac{2}{\pi}\right)^{2 n}
$$

Therefore, we have

$$
\frac{2^{2 n+2}\left(2^{2 n+2}-1\right) B_{n+1}}{(2 n+2) !}<h_{n}(x)<\left(\frac{2}{\pi}\right)^{2 n}
$$

Inequalities in (17) are equivalent to the double inequality (6).

The proof of Theorem 1 is complete.

\section{References}

[1] B.-N. Guo and F. Qi, Generalization of Bernoulli polynomials, Internat. J. Math. Ed. Sci. Tech. 33(2002), 428-431. RGMIA Res. Rep. Coll. 4(2001), Art. 10, 691-695. Available online at http://rgmia.vu.edu.au/v4n4.html

[2] B.-N. Guo, W. Li and F. Qi, Proofs of Wilker's inequalities involving trigonometric functions (On new proofs of inequalities involving trigonometric functions), Inequality Theory and Applications 2(2003), in press. Y. J. Cho, J. K. Kim and S. S. Dragomir (Ed.), Nova Science Publishers. RGMIA Res. Rep. Coll. 3(2000), Art. 15, 167-170. Available online at http://rgmia.vu.edu.au/v3n1.html

[3] S.-L. Guo and F. Qi, Recursion formulae for $\sum_{m=0}^{n} m^{k}$, Z. Anal. Anwendungen 18(1999), 1123-1130.

[4] J.-Ch. Kuang, Chángyòng Bùděngshiì (Applied Inequalities), 2nd ed., Hunan Education Press, Changsha City, China, 1993. (Chinese)

[5] Q.-M. Luo, B.-N. Guo, F. Qi and L. Debnath, Generalizations of Bernoulli's numbers and polynomials, Internat. J. Math. Math. Sci. (2003), in press. RGMIA Res. Rep. Coll. 5(2002), Art. 12, 353-359. Available online at http://rgmia.vu.edu.au/v5n2.html

[6] M. Merkle, Inequalities for residuals of power series: a review, Univ. Beograd. Publ. Elektrotehn. Fak. Ser. Mat. 6(1995), 79-85.

[7] F. Qi. A method of constructing inequalities about $e^{x}$, Univ. Beograd. Publ. Elektrotehn. Fak. Ser. Mat. 8(1997), 16-23.

[8] F. Qi and B.-N. Guo, An upper bound estimate for a complete elliptic integral, Mathematics in Practice and Theory 26(1996), 285-288.

[9] F. Qi and Q.-D. Hao, Refinements and sharpenings of Jordan's and Kober's inequality, Mathematics and Informatics Quarterly 8(1998), 116-120.

[10] F. Qi and Zh. Huang, Inequalities of the complete elliptic integrals, Tamkang J. Math. 29(1998), 165-169.

[11] Zh.-X. Wang and D.-R. Guo, Tèshū Hánshù Gàiùn (Introduction to Special Function), The Series of Adcanced Physics of Peking University, Peking University Press, Beijing, China, 2000. (Chinese)

Department of Applied Mathematics and Informatics, Jiaozuo Institute of Technology, Jiaozuo City, Henan 454000, China.

Department of Applied Mathematics and Informatics, Jiaozuo Institute of Technology, Jiaozuo City, Henan 454000, China.

E-mail: qifeng@jzit.edu.cn or fengqi618@mumber.ams.org

URL: http://rgmia.vu.edu.au/qi.html 